\title{
Predictive Parameters of Decreased Left Ventricular Global Longitudinal Strain at 1 Month After Pediatric Heart Transplantation
}

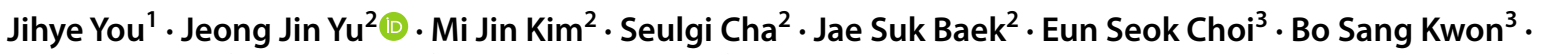 \\ Chun Soo Park ${ }^{3} \cdot$ Tae-Jin Yun $^{3} \cdot$ Young-Hwue Kim²
}

Received: 1 September 2020 / Accepted: 5 January 2021 / Published online: 19 January 2021

(c) The Author(s), under exclusive licence to Springer Science+Business Media, LLC part of Springer Nature 2021

\begin{abstract}
Previous reports indicate that the decreased left ventricular global longitudinal strain (LVGLS) seen in the early postoperative period of pediatric heart transplant patients generally recovers over the course of 1-2 years. In this study, we investigate the predictive capacity of preoperative parameters on the LVGLS decline seen at 1 month post transplant. Forty-six transplant subjects with 2D echocardiographic images sufficient for speckle tracking echocardiography were enrolled. We excluded patients diagnosed with cardiac allograft vasculopathy or with an episode of rejection 1 month before or after their echocardiographic examinations. The mean LVGLS was significantly reduced at 1 month when compared to 1 year following transplant $(-15.5 \%$ vs. $-19.4 \%$, respectively, $p<0.001)$. The predictors of LVGLS that decline at 1 month were the LV mass $z$-score [odds ratio (OR) 1.452; 95\% confidence interval (CI) $1.007-2.095, p=0.046$ ], recipient age (OR 1.124; 95\% CI 1.015-1.245, $p=0.025)$, and donor age (OR 1.081; 95\% CI 1.028-1.136, $p=0.002)$ in the univariate logistic regression analyses. Although multivariate analysis yielded no significant predictors, higher LV mass $z$-scores showed a trend associated with the decline of LVGLS $(p=0.087)$. The donor/recipient weight ratio was associated with the LV mass $z$-score $\left(R^{2}=0.412, p<0.001\right)$.
\end{abstract}

Keywords Echocardiography $\cdot$ Heart transplant $\cdot$ Left ventricular mass $\cdot$ Pediatric $\cdot$ Strain

\section{Introduction}

There are a number of causes of morbidity and mortality post heart transplant including graft dysfunction from allograft rejection, cardiac allograft vasculopathy (CAV), or unknown etiology [1]. Echocardiography is regularly used for monitoring graft function after transplant due to its accessibility and non-invasive nature [2]. Traditionally, left ventricular (LV) fractional shortening and ejection fraction (EF) have been used as measures of global ventricular

Jeong Jin Yu

jjyu@amc.seoul.kr

1 Department of Pediatrics, Sejong General Hospital, Bucheon, Republic of Korea

2 Department of Pediatrics, Asan Medical Center, University of Ulsan College of Medicine, 88, Olympic-ro 43-Gil, Songpa-Gu, Seoul 138-736, Republic of Korea

3 Department of Pediatric Cardiac Surgery, Asan Medical Center, University of Ulsan College of Medicine, Seoul, Republic of Korea function. However, several studies have shown conventional gray-scale echocardiography, including the above volumebased functional variables, to have a low sensitivity for detecting myocardial dysfunction caused by cardiac allograft rejection [3-6]. Other echocardiographic measurements, such as tissue Doppler imaging measurements, are limited by their angle dependence and the translational motion of the heart [7].

Two-dimensional speckle tracking echocardiography is an emerging technique that is reproducible, sensitive, and provides an angle-independent measure of myocardial deformation [strain and strain rate (SR)] [8, 9]. This strain/SR imaging has been reported to be a more sensitive predictor of outcomes than LVEF in diverse clinical situations [6,9]. The utility of strain/SR imaging has been reported in investigations of allograft rejection [6, 10, 11], CAV [12-14], and unknown causes of mortality [15] after heart transplants. However, the interpretation of strain/SR values requires knowledge of the potential range in values under stable conditions, without allograft rejection or CAV. Pediatric heart transplant recipients were reported to have lower LV global 
longitudinal strain (LVGLS) values $[16,17]$ than adult recipients; adult LVGLS values were in the normal range and not different from controls $[18,19]$. Several reports with serial follow-up strain/SR data in pediatric recipients without allograft rejection or CAV showed a pattern in which the LVGLS was decreased at 1 month and recovered over 1-2 years [20,21]. This pattern holds true in our experience, but the results of the measured LVGLS vary on a case-bycase basis. This study aimed to determine predictors of the decreased LVGLS values seen at 1 month after pediatric heart transplantation. A prediction of decreased baseline LVGLS could be expected to improve our understanding of myocardial status during serial follow-up of patients.

\section{Material and Methods}

\section{Subjects}

All consecutive pediatric patients who underwent heart transplantation at Asan Medical Center from January 2007 through December 2017 were considered as subjects for this study. Initially after heart transplant, a "triple therapy" consisting of tacrolimus, mycophenolate mofetil, and prednisolone was attempted for immunosuppression; dose modification or replacement with other drugs was applied in response to adverse reactions or to suppress graft rejection. Regular echocardiographic examinations (including at 1 month and 12 months after transplant) were performed. A routine cardiac biopsy was performed at 1, 2, 3, 6 , and 12 months. Coronary angiography was performed at 1 month and 12 months. Allograft rejection was defined as cellular ( $\geq$ grade $2 \mathrm{R}$ ) or antibody-mediated rejection based on the International Society of Heart and Lung Transplantation (ISHLT) guidelines [22, 23]. When graft rejection was suspected and an augmentation of the immunosuppressive therapy was prompted, allograft rejection was diagnosed regardless of a negative biopsy. Based on the ISHLT guidelines [24], CAV was defined according to the coronary angiography performed at 12 months post transplant.

We included patients with 2D echocardiographic images of sufficient quality for speckle tracking echocardiography obtained at 1 month and 12 months. Demographic and clinical characteristics during the peri-transplantation period were investigated via a review of medical records. B-type natriuretic peptide (BNP) values and the use of antihypertensive medications at each echocardiographic examination were additionally investigated. We excluded patients without available appropriate echocardiographic images, those who were diagnosed with CAV, and those with an episode of rejection (from onset of symptoms through the end of management for rejection) within 1 month before or after echocardiographic examinations. All subjects were divided into two groups of the same number according to LVGLS values 1 month after transplant. Subjects in Group 1 had decreased LVGLS values, and the subjects in Group 2 had relatively preserved values.

This retrospective study was approved by the institutional review board of Asan Medical Center and the requirement for informed patient consent was waived (2020-1375).

\section{Echocardiography}

Echocardiography was conducted using the following machines: Philips IE33 system (Phillips Medical Systems, Andover, MA, USA), General Electric Vivid 7 or E9 machines (GE Health Medical, Horten, Norway), and ACUSON SC2000 (Siemens Medical Solutions USA, Inc., Mountain View, CA, USA). Echocardiographers selected transducers depending on the patient's body size. All echocardiographic data were measured offline and analyzed by an investigator (J.Y.) using TomTec ${ }^{\circledR}$ software (TomTec Imaging Systems; GmbH, Unterschleissheim, Germany). LV mass was calculated on M-mode data using the Devereux equation [25]. The LV mass $z$-score was obtained according to the method outlined by Foster et al. [26].

For the 2D strain analysis, the endocardium was manually traced onto a single frame at end-diastole, after which the strain values were automatically determined by the software. The range of temporal resolution of the stored 2D moving images was 25-102 frames/s. A mean value of two cardiac cycles was used. Longitudinal strain analysis was conducted using apical four-chamber images, and the mean of measured values from six segments was subsequently defined as LVGLS. Circumferential strain analysis was conducted using a parasternal short-axis image at the papillary muscle level, and the mean value of six segments was defined as the LV global circumferential strain. Global longitudinal/circumferential, systolic/diastolic SR were additionally measured in the same way. As a test of reproducibility, 2D moving images from 15 randomly selected subjects were reanalyzed more than 1 week after the initial examination. Another investigator (J.J.Y.) participated in the inter-observer reproducibility testing.

\section{Statistics}

SPSS Statistics for Windows, version 21.0 (IBM Corp., Armonk, NY, USA) was used for statistical analyses. $p$ values $<0.05$ were regarded as statistically significant. Numerical data are presented as mean \pm standard deviation, and categorical data are presented as frequency (\%). BNP values were log-transformed for analysis.

A comparison of echocardiographic data between two time points ( 1 month vs. 12 months after transplant) was performed using a paired $t$ test. Unpaired $t$ tests and Pearson $\chi^{2}$ 
tests were used to compare data between the two groups. To investigate the predictors of decreased LVGLS at 1 month, we performed univariate and multivariate logistic regression analyses with LVGLS treated as a categorical variable (1 for Group 1, and 0 for Group 2). Additionally, linear regression analyses for LVGLS were performed.

\section{Results}

The 46 included patients were divided into two groups based on a cutoff LVGLS value of $-15.5 \%$. Demographic and clinical characteristics are presented in Table 1 . The mean patient age at transplantation was significantly higher in Group 1 than in Group 2 (13.9 vs. 9.5 years, $p=0.019)$. The mean donor age was also significantly higher in Group 1 than in Group 2 (35.8 vs. 19.9 years, $p<0.001)$. The other demographic and clinical variables during the peri-transplantation period were not significantly different between the two groups. An analysis of the level of pulmonary vascular resistance was abandoned due to paucity of data.

One month post operation, the mean BNP levels were not significantly different between groups $(975.0 \pm 783.4 \mathrm{pg} / \mathrm{mL}$ in Group 1 and $701.3 \pm 846.2 \mathrm{pg} /$ $\mathrm{mL}$ in Group 2, $p=0.158$ ), and antihypertensive medications were used by the same number of patients in each group $(n=17,73.9 \%)$.

The incidence of allograft rejection between 2 and 11 months was $17 \%(n=4)$ in Group 1 and $13 \%(n=3)$ in Group $2(p=0.680)$.

After 1 year, the mean BNP levels were not significantly different between the groups $(102.6 \pm 170.6 \mathrm{pg} / \mathrm{mL}$ in Group 1 and 107.1 $\pm 173.2 \mathrm{pg} / \mathrm{mL}$ in Group 2, $p=0.963$ ),
Table 1 Demographic and clinical characteristics during the peri-transplantation period

\begin{tabular}{|c|c|c|c|}
\hline & Group $1(n=23)$ & Group $2(n=23)$ & $p$ value \\
\hline Male gender & $11(48 \%)$ & $16(70 \%)$ & 0.138 \\
\hline Gender concordance between D/R & $13(59 \%)$ & $14(64 \%)$ & 0.757 \\
\hline \multicolumn{4}{|l|}{ Diagnosis } \\
\hline Cardiomyopathy & $16(70 \%)$ & $16(70 \%)$ & \\
\hline Congenital heart diseases & $6(26 \%)$ & $6(26 \%)$ & \\
\hline Re-transplantation & $1(4 \%)$ & $1(4 \%)$ & \\
\hline Age (R) (years) & $13.9 \pm 5.7$ & $9.5 \pm 6.5$ & 0.019 \\
\hline Age (D) (years) & $35.8 \pm 12.2$ & $19.9 \pm 15.4$ & $<0.001$ \\
\hline Body weight (R) (kg) & $41.0 \pm 20.8$ & $33.7 \pm 23.3$ & 0.270 \\
\hline Body weight (D) (kg) & $59.5 \pm 16.0$ & $46.4 \pm 30.1$ & 0.072 \\
\hline $\mathrm{D} / \mathrm{R}$ body weight ratio & $1.76 \pm 0.75$ & $1.48 \pm 0.54$ & 0.147 \\
\hline Listing status ${ }^{\mathrm{a}}$ & & & 0.974 \\
\hline 0 & $10(44 \%)$ & $10(44 \%)$ & \\
\hline 1 & $4(17 \%)$ & $5(22 \%)$ & \\
\hline 2 & $4(17 \%)$ & $4(17 \%)$ & \\
\hline 3 & $5(22 \%)$ & $4(17 \%)$ & \\
\hline Waiting period (days) & $162 \pm 193$ & $378 \pm 648$ & 0.142 \\
\hline \multicolumn{4}{|l|}{ Pre-transplant management } \\
\hline ECMO & $10(44 \%)$ & $9(39 \%)$ & 1.0 \\
\hline Ventilator & $10(44 \%)$ & $11(48 \%)$ & 1.0 \\
\hline Inotropics & $17(74 \%)$ & $19(83 \%)$ & 0.722 \\
\hline Blood type & & & 0.197 \\
\hline A & $3(13 \%)$ & $0(0 \%)$ & \\
\hline B & $7(30 \%)$ & $6(26 \%)$ & \\
\hline $\mathrm{AB}$ & $10(44 \%)$ & $10(44 \%)$ & \\
\hline $\mathrm{O}$ & $3(13 \%)$ & $7(30 \%)$ & \\
\hline Ischemic time (min) & $192 \pm 68$ & $189 \pm 58$ & 0.864 \\
\hline Warm (min) & $74 \pm 25$ & $62 \pm 15$ & 0.046 \\
\hline Cold (min) & $117 \pm 67$ & $113 \pm 59$ & 0.839 \\
\hline
\end{tabular}

$D$ donors, $R$ recipients, $E C M O$ extra-corporeal membrane oxygenation

${ }^{a}$ Classification of listing status for heart transplantation by Korean Centers for Disease Control and Prevention (https://www.konos.go.kr/konosis/common/bizlogic.jsp) 
and the prevalence of antihypertensive medication use was also not significantly different $(n=14,60.9 \%$ in Group 1 vs. $n=8,34.8 \%$ in group $2, p=0.077$ ).

\section{Echocardiography}

Echocardiography findings are presented in Table 2. LV mass $(p<0.001)$ and the LV mass $z$-score $(p<0.001)$ were increased, while LVGLS $(p<0.001)$ and LV global longitudinal SR $(p=0.001)$ were decreased at 1 month when compared to 1 year. These four variables $-\mathrm{LV}$ mass $(p<0.001)$, LV mass $z$-score $(p<0.001)$, LVGLS $(p<0.001)$, and LV global longitudinal SR $(p<0.001)-$ showed the same pattern in the analyses for Group 1, but only LVGLS $(p=0.022)$ significantly recovered at 1 year in Group 2 (Fig. 1).

In the comparisons between the two groups, the LV mass $(p=0.023)$ and the LV mass $z$-score $(p=0.036)$ were higher in Group 1 than Group 2 at 1 month, but they were not different between the groups at 1 year. LVGLS $(p<0.001)$, LV global longitudinal SR $(p<0.001)$, and LV global diastolic longitudinal SR $(p=0.003)$ values were decreased in Group 1 compared to Group 2 at 1 month. LVGLS $(p=0.031)$ and LV global diastolic longitudinal SR $(p=0.047)$ were also decreased in Group 1 compared to Group 2 at 1 year.

There were no significant differences in LV circumferential deformation between either the two time points or the two groups investigated.

\section{Prediction of Decreased LVGLS}

In logistic regression analyses, all demographic and clinical variables in Table 1, as well as echocardiographic results in Table 2, were tested as independent variables for predicting decreased LVGLS at 1 month after transplantation. Recipient age $(p=0.025)$, donor age $(p=0.002)$, and LV mass $z$-score $(p=0.046)$ were significant predictors in the univariate analysis (Table 3 ). Warm ischemic time had a $p$ value $<0.150$ $(p=0.058)$. The log-transformed BNP level was not a significant predictor $(p=0.159)$. In the linear regression analyses, recipient age $\left(R^{2}=0.114, p=0.022\right)$, donor age $\left(R^{2}=0.442\right.$, $p<0.001)$, and LV mass $z$-score $\left(\mathrm{R}^{2}=0.140, p=0.010\right)$ were negatively associated with LVGLS (Fig. 2). There were no significant predictors of reduced LVGLS in the multivariate analyses; however, the LV mass $z$-score trended toward significance $(p=0.087)$.

We performed an additional linear regression analysis for the LV mass $z$-score. Among the demographic and clinical variables collected during the peri-transplantation period, donor/recipient weight ratio $\left(R^{2}=0.412, p<0.001\right)$ and logtransformed BNP level $\left(R^{2}=0.212, p=0.002\right)$ were positively associated with the LV mass $z$-score (Fig. 3).

\section{Reproducibility Testing}

The results of the reproducibility testing for intra-observer and inter-observer variability are presented in Table 4 . The range of intra-class correlation coefficients was 0.806-0.948.

Table 2 Results of echocardiographic examinations at 1 month and 1 year after heart transplantation

\begin{tabular}{|c|c|c|c|c|c|c|}
\hline & \multicolumn{3}{|l|}{ At 1 month } & \multicolumn{3}{|l|}{ At 1 year } \\
\hline & All subjects & Group $1(n=23)$ & Group $2(n=23)$ & All subjects & Group $1(n=23)$ & Group $2(n=23)$ \\
\hline Heart rate (bpm) & $97 \pm 17$ & $93 \pm 17$ & $101 \pm 17$ & $96 \pm 23$ & $89 \pm 15^{\dagger}$ & $103 \pm 28$ \\
\hline \multicolumn{7}{|l|}{ M-mode examination } \\
\hline LVEDD (mm) & $37.7 \pm 8.2$ & $38.5 \pm 7.8$ & $36.9 \pm 8.7$ & $38.7 \pm 6.3$ & $39.1 \pm 5.8$ & $38.3 \pm 7.0$ \\
\hline Fractional shortening (\%) & $37 \pm 6$ & $38 \pm 6$ & $37 \pm 6$ & $39 \pm 8$ & $39 \pm 9$ & $40 \pm 7$ \\
\hline LV mass (g) & $144 \pm 72 *$ & $168 \pm 58 * \dagger$ & $120 \pm 78$ & $117 \pm 55$ & $124 \pm 55$ & $110 \pm 58$ \\
\hline LV mass (z-score) & $1.60 \pm 1.87 *$ & $2.18 \pm 1.83^{* \dagger}$ & $1.03 \pm 1.77$ & $0.50 \pm 1.68$ & $0.29 \pm 1.99$ & $0.70 \pm 1.34$ \\
\hline LVGLS (\%) & $-15.5 \pm 4.1^{*}$ & $-12.1 \pm 2.2 *^{\dagger}$ & $-18.9 \pm 2.6^{*}$ & $-19.4 \pm 4.6$ & $-17.9 \pm 4.5^{\dagger}$ & $-20.8 \pm 4.4$ \\
\hline LVGLSR (\%/s) & $-0.90 \pm 0.28^{*}$ & $-0.70 \pm 0.13 *^{\dagger}$ & $-1.10 \pm 0.24$ & $-1.05 \pm 0.28$ & $-0.98 \pm 0.31$ & $-1.13 \pm 0.23$ \\
\hline LVGLDSR (\%/s) & $0.99 \pm 0.45$ & $0.80 \pm 0.32^{\dagger}$ & $1.18 \pm 0.49$ & $1.11 \pm 0.40$ & $0.99 \pm 0.38^{\dagger}$ & $1.22 \pm 0.40$ \\
\hline LVGCS (\%) & $-24.7 \pm 5.8$ & $-24.3 \pm 6.1$ & $-25.1 \pm 5.6$ & $-25.3 \pm 5.80$ & $-25.9 \pm 6.3$ & $-24.7 \pm 5.4$ \\
\hline LVGCSR (\%/s) & $-1.49 \pm 0.40$ & $-1.50 \pm 0.39$ & $-1.48 \pm 0.43$ & $-1.46 \pm 0.39$ & $-1.50 \pm 0.41$ & $-1.41 \pm 0.38$ \\
\hline LVGCDSR $(\% / s)$ & $1.55 \pm 0.63$ & $1.56 \pm 0.67$ & $1.55 \pm 0.60$ & $1.48 \pm 0.46$ & $1.55 \pm 0.53$ & $1.41 \pm 0.38$ \\
\hline
\end{tabular}

bpm beats per minute, $L V$ left ventricular, $L V E D D$ LV end-diastolic dimension, $L V G L S$ LV global longitudinal strain, $L V G L S R$ LV global longitudinal strain rate, $L V G L D S R$ LV global longitudinal diastolic strain rate, LVGCS LV global circumferential strain, $L V G C S R$ LV global circumferential strain rate, LVGCDSR LV global circumferential diastolic strain rate

$* p<0.05$ in a comparison of data at 1 month and 1 year after heart transplantation

${ }^{\dagger} p<0.05$ in a comparison of data between the two groups 

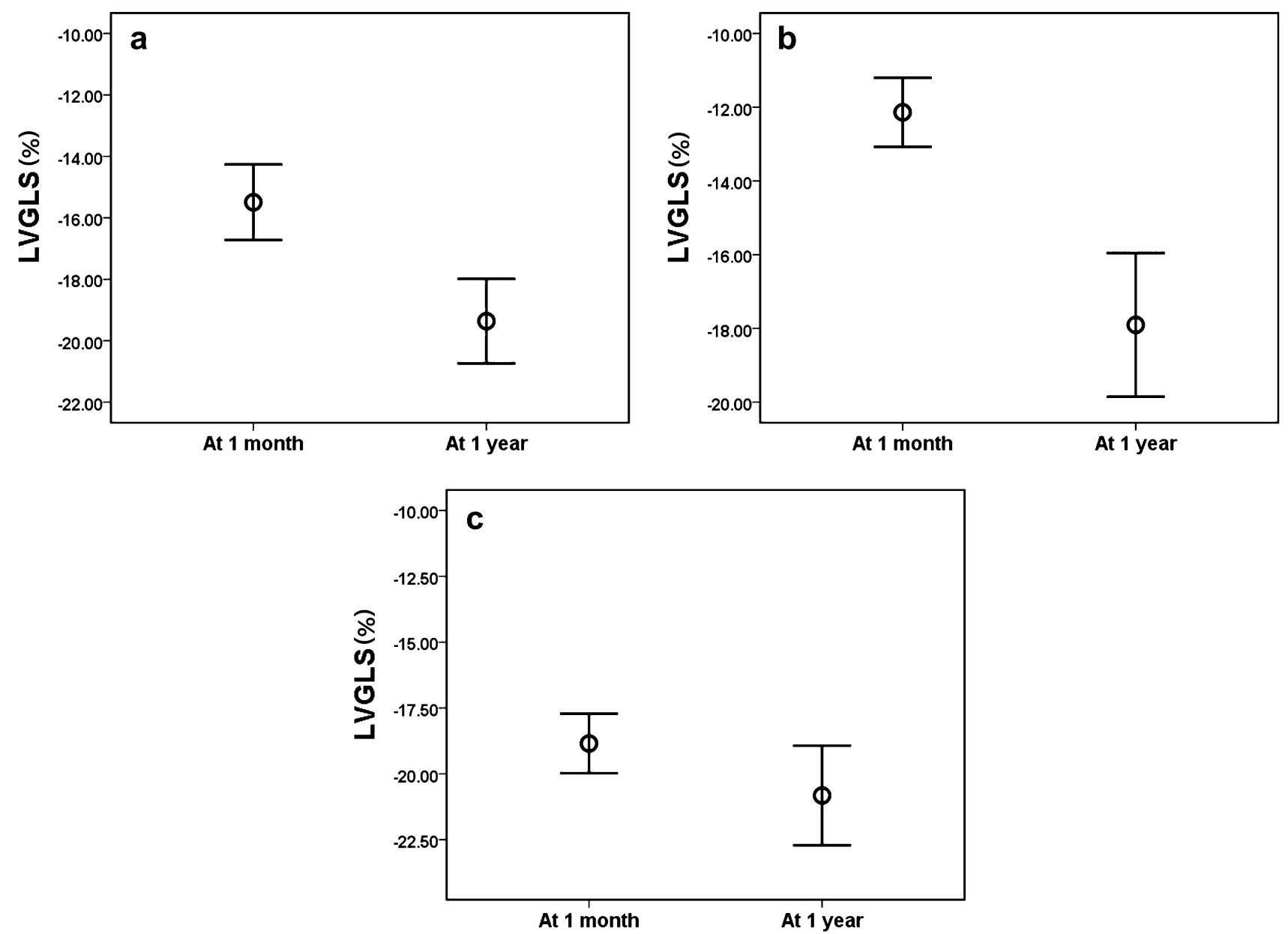

Fig. 1 A comparison of left ventricular global longitudinal strain (LVGLS) values measured at 1 month and 1 year after heart transplantation in all subjects (a), Group 1 (b), and Group 2 (c). The

mean LVGLS was higher at 1 year than at 1 month in all analyses $(p<0.001, p<0.001$, and $p=0.027$, respectively)

heart transplantation. The LV global longitudinal SR showed the same pattern. These results are consistent with recent reports [20,21]. After 1 year, the LVGLS of all subjects was recovered to $-19.4 \%$, within the normal range of non-transplanted hearts of -18.6 to $-22.5 \%$ [27-29]. Univariate logistic regression analyses revealed LV mass $z$-score, donor age, and recipient age as significant predictors of decreased LVGLS at 1 month; these variables were also significantly associated with LVGLS in the linear regression analyses. Although these variables were not significant predictors in the multivariate analysis, higher LV mass $z$-score showed a trend associated with the decline of LVGLS ( $p=0.087)$.

It has been reported that the increase in LV mass initially following transplantation normalized over 6 months to 2 years along with the graft remodeling in the context of pediatric heart transplantation [21, 30]. In our study, we observed the same pattern of changes in LV mass following transplantation. This pattern of temporal change in the LV 

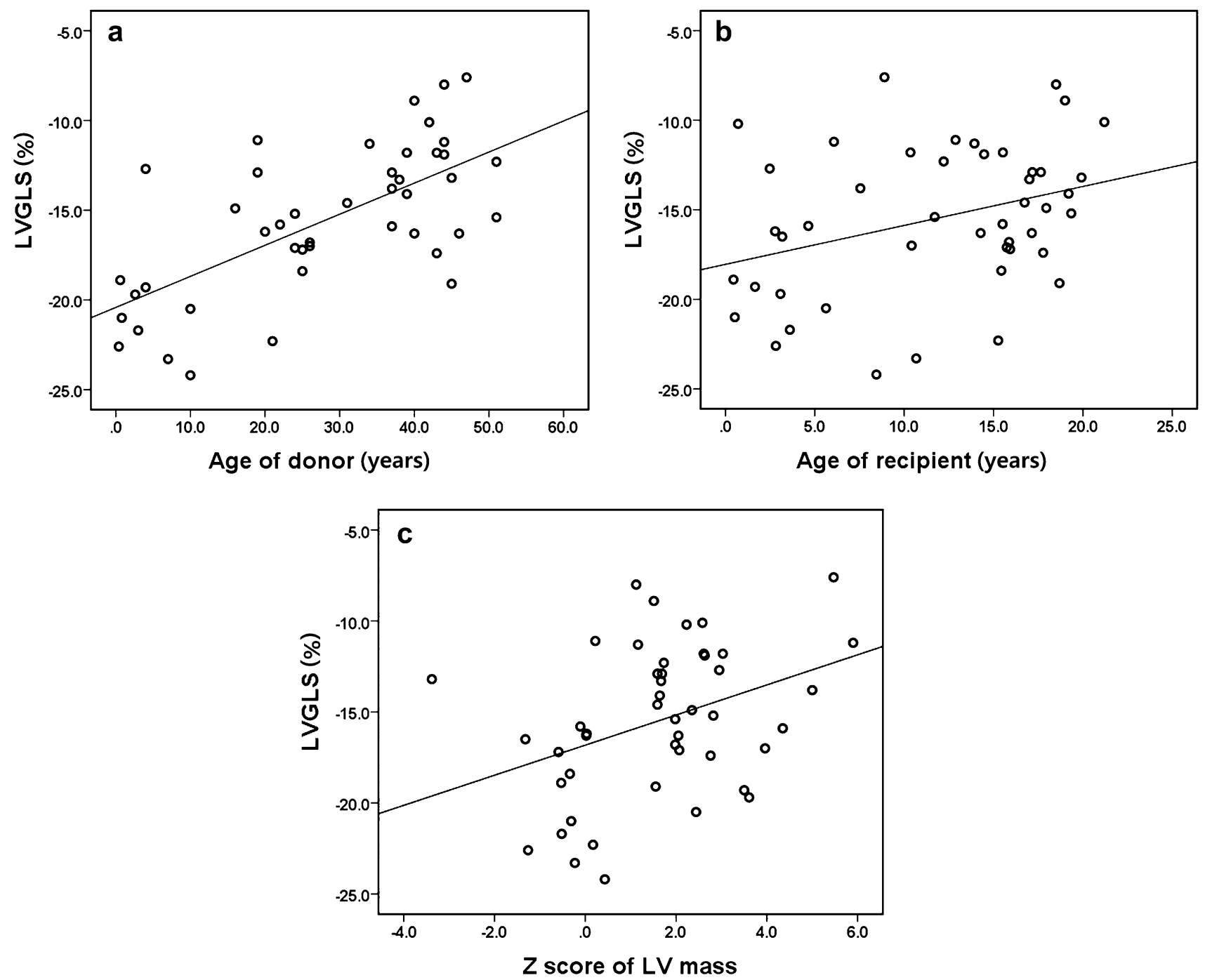

Fig. 2 Results of linear regression analysis using left ventricular global longitudinal strain (LVGLS) as a dependent variable. The donor age $\left(R^{2}=0.442, p<0.001\right)$, the recipient age $\left(R^{2}=0.114\right.$,

mass could be a result of the LV adaptation process following transplantation. It is possible that the increase in LV mass contributes to the decrease in LVGLS at 1 postoperative month. Decreased LVGLS in LV hypertrophy has been reported in diverse cardiac illnesses including hypertrophic cardiomyopathy, uremia, systemic hypertension, and aortic stenosis [31-34].

In linear regression analysis of the LV mass $z$-score, donor/recipient weight ratio and log-BNP level were found to be associated. A higher donor/recipient weight ratio means that the donor heart size is large relative to that of the recipient. Therefore, a higher donor/recipient weight ratio might contribute to an increased LV mass $z$-score at 1 month. However, with regard to the association between LV mass $z$-score and log-BNP level, it seems reasonable to $p=0.022)$, and the LV $z$-score $\left(R^{2}=0.140, p=0.010\right)$ measured at 1 month after transplantation were significant independent predictors of decreased LVGLS

think that the alterations of these variables may both result from other stress factors, because LV hypertrophy generally induces a reduction of BNP level.

In this study, the higher ages of recipients and donors were also significant predictors of decreased LVGLS at 1 month following transplantation. However, it is difficult to further explain the role of age as an etiologic factor for LVGLS decline solely using the results of this study.

Wisotzkey et al. reported that higher log-BNP levels were associated with decreased LVGLS following pediatric heart transplant [17]. In their study, the data collection period was not specified. However, log-BNP level was not a significant predictor of LVGLS decline at 1 month in this study. It was reported that increased serum BNP levels from the early post-transplantation period slowly improved [35]. In this 


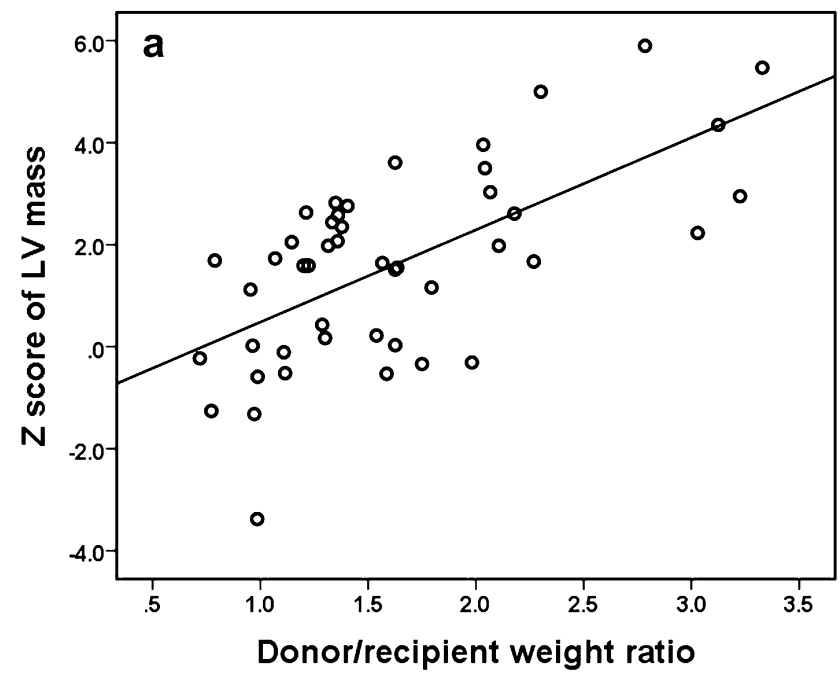

Fig. 3 Results of linear regression analysis using the LV mass $z$-score at 1 month as a dependent variable. The donor/recipient weight ratio $\left(R^{2}=0.412, p<0.001\right)$ (a) and the log-transformed value of the

Table 4 Intra-class correlation coefficients in reproducibility testing for the measurement of left ventricular deformation

\begin{tabular}{lll}
\hline & Intra-observer & Inter-observer \\
\hline LVGLS $(\%)$ & 0.806 & 0.853 \\
LVGLSR $(\% / s)$ & 0.835 & 0.90 \\
LVGLDSR $(\% / s)$ & 0.948 & 0.942 \\
LVGCS $(\%)$ & 0.943 & 0.839 \\
LVGCSR $(\% / s)$ & 0.942 & 0.850 \\
LVGCDSR $(\% / s)$ & 0.939 & 0.920 \\
\hline
\end{tabular}

LVGLS LV global longitudinal strain, LVGLSR LV global longitudinal strain rate, $L V G L D S R$ LV global longitudinal diastolic strain rate, LVGCS LV global circumferential strain, LVGCSR LV global circumferential strain rate, $L V G C D S R$ LV global circumferential diastolic strain rate

study, serum BNP level was obtained at 1 month when they would be unstable.

In this study, LV circumferential deformation measurements were relatively preserved at 1 month post transplant and showed no difference between the two time points investigated. The phenomenon of discrepant results between longitudinal and circumferential strain has been observed previously $[20,21]$. The presumptive causes for this phenomenon are the susceptibility of subendocardial longitudinal myocardial fibers to ischemic damage and a greater functional reserve of circumferential fibers [20]. During heart transplantation surgery, exposure to some degree of ischemia is inevitable. The circumferential fibers, which are predominantly in the middle myocardial layer, are less susceptible to ischemic damage [36, 37]. In this study, the mean warm ischemic time was longer in

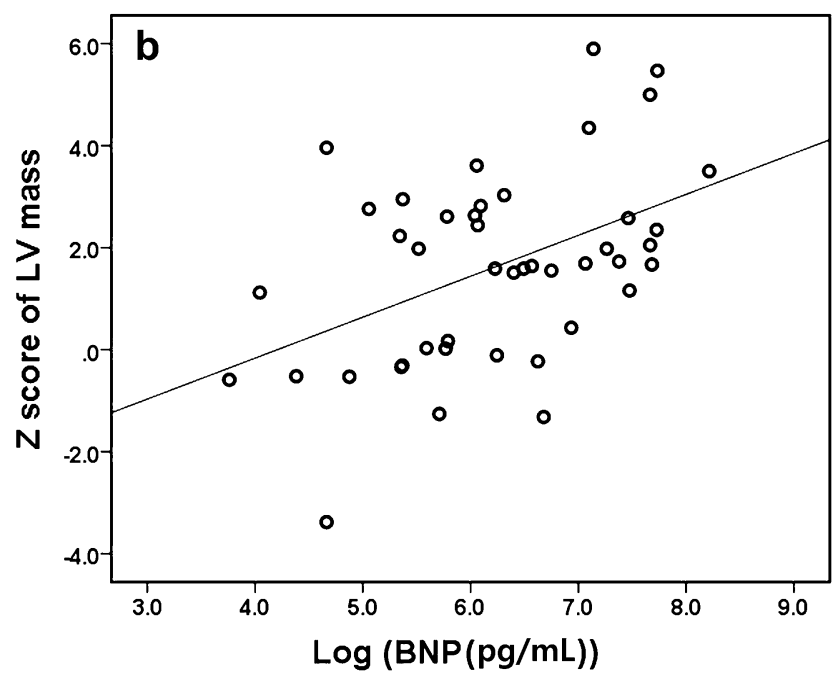

B-type natriuretic peptide (BNP) level $\left(R^{2}=0.212, p=0.002\right)$ (b) were significantly associated with LV mass $z$-score

Group 1 (with relatively decreased LVGLS) than in Group 2 , but it was not significantly associated with LVGLS decline in the regression analyses.

\section{Study Limitations}

This retrospective, single-center study had several limitations. First, for the donors, only demographic data were available. Although the donor age was a significant predictor for LVGLS decline 1 month after transplantation in the univariate analysis, no further investigation was possible in this regard. Additional donor data, such as the cause of brain death and the details of intensive care management before organ harvesting, may have been helpful. Second, the small sample size may have contributed to the lack of statistically significant results yielded by the multivariate logistic regression analysis.

\section{Conclusions}

LV mass $z$-score, donor age, and recipient age were significant predictors of decreased LVGLS 1 month after transplantation in the univariate analysis. Although multivariate analysis yielded no significant predictors, higher LV mass $z$-score showed a trend associated with the decline of LVGLS $(p=0.087)$. LV mass $z$-score was associated with the donor/recipient weight ratio. 
Data Availability Investigation of data for this study was conducted with the approval of the institutional review board of the Asan Medical Center, Seoul, Republic of Korea.

Code Availability TomTec ${ }^{\circledR}$ software (TomTec Imaging Systems; $\mathrm{GmbH}$, Unterschleissheim, Germany) was used for echocardiographic analyses. SPSS Statistics for Windows, version 21.0 (IBM Corp., Armonk, NY, USA) was used for statistical analyses. Both software applications were legally acquired by Asan Medical Center for use by hospital researchers.

\section{Compliance with Ethical Standards}

Conflict of Interest There are no conflicts of interest or competing interests.

\section{References}

1. Shahzad K, Aziz QA, Leva JP et al (2011) New-onset graft dysfunction after heart transplantation-incidence and mechanism related outcomes. J Heart Lung Transplant 30:194-203

2. Valantine HA, Appleton CP, Hatle LK et al (1989) A hemodynamic and Doppler echocardiographic study of ventricular function in long-term cardiac allograft recipients. Etiology and prognosis of restrictive-constrictive physiology. Circulation 79:66-75

3. Frommelt MA, Snider AR, Crowley DC et al (1992) Echocardiographic indexes of allograft rejection in pediatric cardiac transplant recipients. J Am Soc Echocardiogr 5:41-47

4. Hsu DT, Sponitz HM (1990) Echocardiographic diagnosis of cardiac allograft rejection. Prog Cardiovasc Dis 33:149-160

5. Streeter RP, Nichols K, Bergmann SR (2005) Stability of right and left ventricular ejection fractions and volumes after heart transplantation. J Heart Lung Transplant 24:815-818

6. Marciniak A, Eroglu E, Marciniak M et al (2007) The potential clinical role of ultrasonic strain and strain rate imaging in diagnosing acute rejection after heart transplantation. Eur J Echocardiogr 3:213-221

7. Mor-Avi V, Lang RM, Badano LP et al (2011) Current and evolving echocardiographic techniques for the quantitative evaluation of cardiac mechanics: ASE/EAE consensus statement on methodology and indications endorsed by the Japanese Society of Echocardiography. Eur J Echocardiogr 12:167-205

8. Dandel M, Hetzer R (2009) Echocardiographic strain and strain rate imaging-clinical applications. Int J Cardiol 132:11-24

9. Zhang KW, French B, May Khan A et al (2014) Stain improves risk prediction beyond ejection fraction in chronic systolic heart failure. J Am Heart Assoc 3:e000550

10. Mingo-Santos S, Monivas-Palomero V, Garcia-Lunar I et al (2015) Usefulness of two-dimensional strain parameters to diagnose acute rejection after heart transplantation. J Am Soc Echocardiogr 28:1149-1156

11. Podrouzkova H, Bedanova H, Tretina M et al (2015) Decrease in longitudinal strain in heart transplant recipients is associated with rejection. Biomed Pap Med Fac Univ Palacky Olomouc Czech Repub 159:601-606

12. Clemmensen TS, Logstrup BB, Eiskjaer H et al (2015) Evaluation of longitudinal myocardial deformation by 2-dimensional speckle-tracking echocardiography in heart transplant recipients: relation to coronary allograft vasculopathy. J Heart Lung Transplant 34:195-203

13. Zoeller BB, Miyamoto SD, Younoszai AK et al (2016) Longitudinal strain and strain rate abnormalities precede invasive diagnosis of transplant coronary artery vasculopathy in pediatric cardiac transplant patients. Pediatr Cardiol 37:656-662

14. Boruta RJ, Miyamoto SD, Younoszai AK et al (2018) Worsening in longitudinal strain and strain rate anticipates development of pediatric transplant coronary artery vasculopathy as soon as one year following transplant. Pediatr Cardiol 39:129-139

15. Sarvari SI, Gjesdal O, Gude E et al (2012) Early postoperative left ventricular function by echocardiographic strain is a predictor of 1-year mortality in heart transplant recipients. J Am Soc Echocardiogr 25:1007-1014

16. Kailin JA, Miyamoto SD, Younoszai AK et al (2012) Longitudinal myocardial deformation is selectively decreased after pediatric cardiac transplantation: a comparison of children 1 year after transplantation with normal subjects using velocity vector imaging. Pediatr Cardiol 33:749-756

17. Wisotzkey BL, Jorgensen NW, Albers EL et al (2017) Feasibility and interpretation of global longitudinal strain imaging in pediatric heart transplant recipients. Pediatr Transplant 21:e12909

18. Pichler P, Binder T, Höfer P et al (2012) Two-dimensional speckle tracking echocardiography in heart transplant patients: three-year follow-up of deformation parameters and ejection fraction derived from transthoracic echocardiography. Eur Heart J Cardiovasc Imaging 13:181-186

19. Palomero VM, Santos SM, Artaza JG et al (2016) Two-dimensional speckle tracking echocardiography in heart transplant patients: two-year follow-up of right and left ventricular function. Echocardiography 33:703-713

20. Godown J, Dodd DA, Stanley M et al (2018) Changes in left ventricular strain parameters following pediatric heart transplantation. Pediatr Transplant 22:e13166

21. Sahawalla R, Sehgal S, Blake J et al (2019) Left ventricular adaptation following orthotopic heart transplantation in children: a speckle tracking echocardiographic imaging study. Clin Transplant 33:e13632

22. Stewart S, Winters GL, Fishbein MC et al (2005) Revision of the 1990 working formulation for the standardization of nomenclature in the diagnosis of heart rejection. J Heart Lung Transplant 24:1710-1720

23. Berry GJ, Burke MM, Andersen C et al (2013) The 2013 international society for heart and lung transplantation working formulation for the standardization of nomenclature in the pathologic diagnosis of antibody-mediated rejection in heart transplantation. J Heart Lung Transplant 32:1147-1162

24. Mehra MR, Crespo-Leiro MG, Dipchand A et al (2010) International Society for Heart and Lung Transplantation working formulation of a standardized nnomenclature for cardiac allograft vasculopathy. J Heart Lung Transplant 29:717-727

25. Devereux RB, Alonso DR, Lutas EM et al (1986) Echocardiographic assessment of left ventricular hypertrophy: comparison to necropsy findings. Am J Cardiol 57:450-458

26. Foster BJ, Mackie AS, Mitsnefes M et al (2008) A novel method of expressing left ventricular mass relative to body size in children. Circulation 117:2275-2769

27. Marwick TH, Leano RL, Brown J et al (2009) Myocardial strain measurement with 2-dimensional speckle-tracking echocardiography: definition of normal range. JACC Cardiovasc Imaging 2:80-84

28. Marcus KA, Mavinkurve-Groothuis AMC, Barends M et al (2011) Reference values for myocardial two-dimensional strain echocardiography in a healthy pediatric and young adult cohort. J Am Soc Echocardiogr 24:625-636

29. Kaku K, Takeuchi M, Tsang W et al (2014) Age-related normal range of left ventricular strain and torsion using three-dimensional speckle-tracking echocardiography. J Am Soc Echocardiogr 27:55-64 
30. Shirali GS, Lombano F, Lawrence Beeson W et al (1995) Ventricular remodeling following infant-pediatric cardiac transplantation. Does age at transplantation or size disparity matter? Transplantation 60:1467-1472

31. Schnell F, Donal E, Bernard-Brunet A et al (2013) Strain analysis during exercise in patients with left ventricular hypertrophy: impact of etiology. J Am Soc Echocardiogr 26:1163-1169

32. Yi Z, Yang X, Zhou Q et al (2017) Effect of left ventricular hypertrophy and deformation on cardiac function in patients with uremia cardiomyopathy by using quantitative speckle tracking technology. Zhong Nan Da Xue Xue Bao Yi Xue Ban 42:400-405

33. Sun JP, Xu TY, Ni XD et al (2019) Echocardiographic strain in hypertrophic cardiomyopathy and hypertensive left ventricular hypertrophy. Echocardiography 36:257-265

34. Vigneault DM, Yang E, Jensen PJ et al (2019) Left ventricular strain in abnormal in preclinical and overt hypertrophic cardiomyopathy: cardiac MR feature tracking. Radiology 290:640-648

35. Garrido IP, Pascual-Figal DA, Nicolas F et al (2009) Usefulness of serial monitoring of B-type natriuretic peptide for the detection of acute rejection after heart transplantation. Am J Cardiol 103:1149-1153

36. Greenbaum RA, Ho SY, Gibson DG et al (1981) Left ventricular fibre architecture in man. Br Heart J 45:248-263

37. Spotnitz HM (2000) Macro design, structure, and mechanics of the left ventricle. J Thorac Cardiovasc Surg 119:1053-1077

Publisher's Note Springer Nature remains neutral with regard to jurisdictional claims in published maps and institutional affiliations. 